\title{
Heine's basic transform and a permutation group for $q$-harmonic series
}

by

\section{WADiM Zudilin (Moscow)}

1. Introduction. More than 150 years ago, E. Heine [He1], [He2] considered the series

$$
\begin{aligned}
&{ }_{2} \phi_{1}\left(\begin{array}{r}
\left.q^{a}, q^{b} \mid q, z\right)=1 \\
q^{c} \mid q,
\end{array}\right. \frac{\left(1-q^{a}\right)\left(1-q^{b}\right)}{(1-q)\left(1-q^{c}\right)} z \\
&+\frac{\left(1-q^{a}\right)\left(1-q^{a+1}\right)\left(1-q^{b}\right)\left(1-q^{b+1}\right)}{(1-q)\left(1-q^{2}\right)\left(1-q^{c}\right)\left(1-q^{c+1}\right)} z^{2}+\ldots, \\
&|q|<1, \quad|z|<1, \quad c \neq 0,-1,-2, \ldots,
\end{aligned}
$$

and proved several results for it. In particular, he obtained the transformation formula

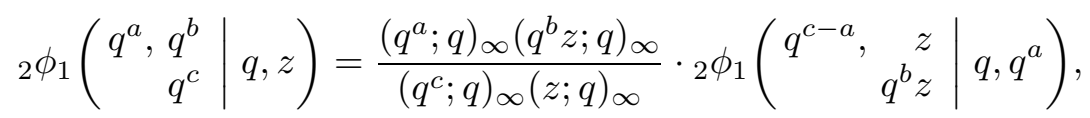

where $(x ; q)_{\infty}:=\prod_{n=1}^{\infty}\left(1-x q^{n-1}\right)$. Today generalized Heine series become an actively investigated part of modern mathematics and a lot of papers and monographs (see, e.g., [Ex], [Fi], [GR]) are devoted to their study. The aim of this note is to make use of Heine's transform (1) to deduce a sharp irrationality measure for the $q$-harmonic series

$$
\begin{aligned}
& h_{p}(1):=\sum_{n=1}^{\infty} \frac{1}{p^{n}-1}=\sum_{n=1}^{\infty} \frac{q^{n}}{1-q^{n}}
\end{aligned}
$$

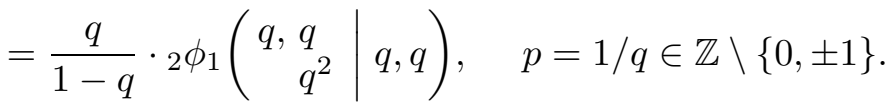

As is easily seen, the series $h_{p}(1)$ is irrational as a function of $p$. The irrationality of the number $h_{2}(1)$ (i.e., $q=1 / 2$ in (2)) was first proved by P. Erdős [Er], who also posed the problem of extending his result to an arbitrary integer $p \in \mathbb{Z} \backslash\{0, \pm 1\}$. This problem (even in more general

2000 Mathematics Subject Classification: 11J82, 33D15. 
settings) was solved by J.-P. Bézivin [Bé] and in a quantitative form by P. Borwein $[\mathrm{Bo}]$. A sharp irrationality measure

$$
\mu\left(h_{p}(1)\right) \leq \frac{2 \pi^{2}}{\pi^{2}-2}=2.50828476 \ldots
$$

was obtained by P. Bundschuh and K. Väänänen [BV] with the help of explicit Padé approximations to the $q$-logarithm function and a certain $q$ arithmetic observation. (Here $\mu=\mu(\alpha)$ denotes the irrationality exponent of an irrational number $\alpha$ that is the least possible exponent such that for any $\varepsilon>0$ the inequality $|\alpha-a / b| \leq b^{-(\mu+\varepsilon)}$ has only finitely many solutions in integers $a, b$.) The works [BV], [MV], and [Ass] contain estimates for irrationality exponents of the $q$-logarithm values. Finally, introducing a $q$-arithmetic approach in [Zu1] resulted in a slight improvement of the estimate (3) and of the estimate in [Ass] for a $q$-analogue of $\log 2$ (the result for $\mu\left(h_{p}(1)\right)$ in [Zu1] is wrong due to a computational error; see the remark at the end of Section 3 below).

The "ordinary" arithmetic approach occurs as a part of the groupstructure approach proposed by G. Rhin and C. Viola in [RV1], [RV2] for obtaining quantitative results for the values $\zeta(2)$ and $\zeta(3)$ of Riemann's zeta function. Recently, the author [Zu2] extended the method of [RV1] to a suitable $q$-analogue of $\zeta(2)$. The permutation group in [RV1], [Zu2] is rather rich to get nice estimates for irrationality exponents in both ordinary and $q$-(basic) cases. A simpler group (of order 12) for the $q$-harmonic series that appears below also leads to a quantitative result.

THEOREM 1. The irrationality exponent of $h_{p}(1)$ satisfies the estimate

$$
\mu\left(h_{p}(1)\right) \leq 2.46497868 \ldots
$$

However, the group has no ordinary analogue, hence it has not appeared before in an arithmetic study. As pointed out by the referee, it is worth remarking that the estimate in Theorem 1 is uniform in $p$.

2. $q$-Basis. Throughout the paper $p=1 / q \in \mathbb{Z} \backslash\{0, \pm 1\}$. As usual (see, e.g., $[\mathrm{GR}])$, define the shifted q-factorial

$$
(a ; q)_{0}=1, \quad(a ; q)_{n}:=\prod_{\nu=1}^{n}\left(1-a q^{\nu-1}\right) \quad \text { for } n=1,2, \ldots,
$$

and Jackson's q-gamma function

$$
\Gamma_{q}(t):=\frac{(q ; q)_{\infty}}{\left(q^{t} ; q\right)_{\infty}}(1-q)^{1-t} .
$$

Then $q$-extensions of factorial and binomial coefficients read as follows:

$$
[n]_{q} !:=\Gamma_{q}(n+1)=\frac{(q ; q)_{n}}{(1-q)^{n}}=p^{-n(n-1) / 2}[n]_{p} !,
$$




$$
\left[\begin{array}{l}
n \\
k
\end{array}\right]_{q}:=\frac{(q ; q)_{n}}{(q ; q)_{k} \cdot(q ; q)_{n-k}}=\frac{[n]_{q} !}{[k]_{q} ![n-k]_{q} !}=p^{-k(n-k)}\left[\begin{array}{c}
n \\
k
\end{array}\right]_{p},
$$

where $k=0,1, \ldots, n$ and $n=0,1,2, \ldots$

Since $p^{n}-1=\prod_{l \mid n} \Phi_{l}(p)$, where

$$
\Phi_{l}(p):=\prod_{\substack{k=1 \\(k, l)=1}}^{l}\left(p-e^{2 \pi i k / l}\right) \in \mathbb{Z}[p], \quad l=1,2,3, \ldots,
$$

are (irreducible over $\mathbb{Z}$ ) cyclotomic polynomials, we deduce the following claims:

- the polynomials (4) are the only irreducible divisors of the polynomial

$$
[n]_{p} !=\prod_{\nu=1}^{n} \frac{p^{\nu}-1}{p-1} \in \mathbb{Z}[p]
$$

moreover,

$$
\operatorname{ord}_{\Phi_{1}(p)}[n]_{p} !=0, \quad \operatorname{ord}_{\Phi_{l}(p)}[n]_{p} !=\left\lfloor\frac{n}{l}\right\rfloor, \quad l=2,3,4, \ldots,
$$

where $\lfloor\cdot\rfloor$ denotes the integral part of a number;

- the polynomial $D_{n}(p):=\prod_{l=1}^{n} \Phi_{l}(p)$ is the least common multiple of the polynomials $p^{k}-1, k=1, \ldots, n$.

In the above notation, the $q$-arithmetic approach is characterized by the following assertions.

Lemma 1 ([BV, Section 2]; [Ass, Lemma 2]). We have

$$
\lim _{n \rightarrow \infty} \frac{\log \left|D_{n}(p)\right|}{n^{2} \log |p|}=\frac{3}{\pi^{2}}
$$

Lemma 2 ([Zu1, Lemma 1$])$. For each demi-interval $[u, v) \subset(0,1)$ with $u, v \in \mathbb{Q}$,

$$
\begin{aligned}
\lim _{n \rightarrow \infty} \frac{1}{n^{2} \log |p|} \sum_{l:\{n / l\} \in[u, v)} \log \left|\Phi_{l}(p)\right| & =\frac{3}{\pi^{2}}\left(\psi^{\prime}(u)-\psi^{\prime}(v)\right) \\
& =\frac{3}{\pi^{2}} \int_{u}^{v} d\left(-\psi^{\prime}(x)\right),
\end{aligned}
$$

where $\{a\}=a-\lfloor a\rfloor$ and $\psi(x)$ is the logarithmic derivative of Euler's gamma function.

3. Linear forms involving $q$-harmonic series. Let $a_{0}, a_{1}, a_{2}$, and $b$ be positive integers satisfying the condition

$$
a_{1}+a_{2} \leq b \text {. }
$$


Consider the rational function

$$
R(T)=\frac{(q T ; q)_{a_{1}-1}}{(q ; q)_{a_{1}-1}} \cdot \frac{(q ; q)_{b-a_{2}-1}}{\left(q^{a_{2}} T ; q\right)_{b-a_{2}-1}} \cdot T^{a_{0}},
$$

so that

$$
R\left(q^{t}\right)=\frac{\Gamma_{q}\left(b-a_{2}\right)}{(1-q) \Gamma_{q}\left(a_{1}\right)} \cdot \frac{\Gamma_{q}\left(t+a_{1}\right) \Gamma_{q}\left(t+a_{2}\right)}{\Gamma_{q}(t+1) \Gamma_{q}(t+b)} \cdot q^{a_{0} t} .
$$

Denote by $a_{1}^{*} \leq a_{2}^{*}$ the ordered version of the set $a_{1}, a_{2}$, i.e., $\left\{a_{1}^{*}, a_{2}^{*}\right\}=$ $\left\{a_{1}, a_{2}\right\}$. Condition (6) implies that $R(T) T^{-a_{0}}=O\left(T^{-1}\right)$ as $T \rightarrow \infty$, hence we can write the partial-fraction decomposition

where

$$
R(T)=T^{a_{0}} \sum_{k=a_{2}^{*}}^{b-1} \frac{A_{k}}{1-q^{k} T},
$$

$$
\begin{aligned}
A_{k}= & \left.\left(R(T) T^{-a_{0}}\left(1-q^{k} T\right)\right)\right|_{T=q^{-k}} \\
= & (-1)^{a_{1}+a_{2}+k+1} q^{\left(a_{1}-2 k\right)\left(a_{1}-1\right) / 2+\left(k-a_{2}\right)\left(k-a_{2}+1\right) / 2} \\
& \times\left[\begin{array}{c}
k-1 \\
a_{1}-1
\end{array}\right]_{q}\left[\begin{array}{c}
b-a_{2}-1 \\
b-k-1
\end{array}\right]_{q} \\
= & (-1)^{a_{1}+a_{2}+k+1} p^{a_{1}\left(a_{1}-1\right) / 2-\left(b-a_{2}\right)\left(b-a_{2}-1\right) / 2+(b-k)(b-k-1) / 2} \\
& \times\left[\begin{array}{c}
k-1 \\
a_{1}-1
\end{array}\right]_{p}\left[\begin{array}{c}
b-a_{2}-1 \\
b-k-1
\end{array}\right]_{p} .
\end{aligned}
$$

Furthermore, consider the series

$$
\begin{aligned}
F(\boldsymbol{a} ; b) & =F\left(a_{0}, a_{1}, a_{2} ; b\right):=\sum_{t=0}^{\infty} R\left(q^{t}\right)=\sum_{t=1-a_{1}^{*}}^{\infty} R\left(q^{t}\right) \\
& =\sum_{k=a_{2}^{*}}^{b-1} A_{k} \sum_{t=1-a_{1}^{*}}^{\infty} \frac{q^{a_{0} t}}{1-q^{k+t}}=\sum_{k=a_{2}^{*}}^{b-1} A_{k} q^{-a_{0} k} \sum_{l=k-a_{1}^{*}+1}^{\infty} \frac{q^{a_{0} l}}{1-q^{l}} \\
& =\sum_{k=a_{2}^{*}}^{b-1} A_{k} p^{a_{0} k}\left(\sum_{l=1}^{\infty} \frac{q^{l}}{1-q^{l}}-\sum_{l=1}^{k-a_{1}^{*}} \frac{q^{l}}{1-q^{l}}-\sum_{l=k-a_{1}^{*}+1}^{\infty} \frac{q^{l}-q^{a_{0} l}}{1-q^{l}}\right) \\
& =A(p) h_{p}(1)-B_{1}(p)-B_{2}(p),
\end{aligned}
$$

where

$$
A(p)=\sum_{k=a_{2}^{*}}^{b-1} A_{k} p^{a_{0} k}, \quad B_{1}(p)=\sum_{k=a_{2}^{*}}^{b-1} A_{k} p^{a_{0} k} \sum_{l=1}^{k-a_{1}^{*}} \frac{1}{p^{l}-1}
$$

and 


$$
\begin{aligned}
B_{2}(p) & =\sum_{k=a_{2}^{*}}^{b-1} A_{k} p^{a_{0} k} \sum_{l=k-a_{1}^{*}+1}^{\infty} \sum_{j=1}^{a_{0}-1} q^{j l} \\
& =\sum_{k=a_{2}^{*}}^{b-1} A_{k} p^{a_{0} k} \sum_{j=1}^{a_{0}-1} \frac{\left(q^{j}\right)^{k-a_{1}^{*}+1}}{1-q^{j}} \\
& =\sum_{k=a_{2}^{*}}^{b-1} \sum_{j=1}^{a_{0}-1} \frac{A_{k} p^{a_{0} k-j\left(k-a_{1}^{*}\right)}}{p^{j}-1} .
\end{aligned}
$$

Now, note that $a_{0} k \geq a_{0} a_{2}^{*}$ and $a_{0} k-j\left(k-a_{1}^{*}\right)>a_{0} k-a_{0}\left(k-a_{1}^{*}\right)=a_{0} a_{1}^{*}$ for $a_{2}^{*} \leq k<b, 1 \leq j<a_{0}$. Hence, taking

$$
M_{0}=a_{0} a_{1}^{*}+a_{1}\left(a_{1}-1\right) / 2-\left(b-a_{2}\right)\left(b-a_{2}-1\right) / 2,
$$

from (8) we deduce the inclusions

$$
p^{-M_{0}} A_{k} p^{a_{0} k} \in \mathbb{Z}[p], \quad p^{-M_{0}} A_{k} p^{a_{0} k-j\left(k-a_{1}^{*}\right)} \in \mathbb{Z}[p] .
$$

Applying the results of Section 2 to formulae (10) and (11) we obtain

$$
\begin{gathered}
p^{-M_{0}} A(p) \in \mathbb{Z}[p], \\
p^{-M_{0}} D_{b-a_{1}^{*}-1}(p) B_{1}(p) \in \mathbb{Z}[p], \quad p^{-M_{0}} D_{a_{0}-1}(p) B_{2}(p) \in \mathbb{Z}[p] .
\end{gathered}
$$

Therefore representation (9) yields the following assertion.

LEMMA 3. With a suitable integer

$$
M=M(\boldsymbol{a} ; b) \geq a_{0} a_{1}^{*}+\frac{a_{1}\left(a_{1}-1\right)}{2}-\frac{\left(b-a_{2}\right)\left(b-a_{2}-1\right)}{2}
$$

we have the inclusion

$$
p^{-M} D_{\max \left\{a_{0}-1, b-a_{1}^{*}-1\right\}}(p) F(\boldsymbol{a} ; b) \in \mathbb{Z}[p] h_{p}(1)+\mathbb{Z}[p] .
$$

Thanks to $(7)$, the quantity $F(\boldsymbol{a} ; b)$ can be identified with the Heine series:

$$
F\left(a_{0}, a_{1}, a_{2} ; b\right)=\frac{\Gamma_{q}\left(a_{2}\right) \Gamma_{q}\left(b-a_{2}\right)}{(1-q) \Gamma_{q}(b)} \cdot{ }_{2} \phi_{1}\left(\begin{array}{c|c}
q^{a_{1}}, q^{a_{2}} & q, q^{a_{0}} \\
q^{b} &
\end{array}\right) .
$$

The lower estimate in $(12)$ for $M(\boldsymbol{a} ; b)$ is rather rough and we require the following sharp form of it.

Lemma 4. Suppose that

$$
a_{1} \leq a_{2}, \quad a_{1}+a_{2} \leq b \leq a_{0}+a_{2} .
$$

Then

$$
p^{-M} D_{\max \left\{a_{0}-1, b-a_{1}-1\right\}}(p) F(\boldsymbol{a} ; b) \in \mathbb{Z}[p] h_{p}(1)+\mathbb{Z}[p],
$$

where

$$
M=M(\boldsymbol{a} ; b):=\frac{a_{1}\left(a_{1}-1\right)}{2}+a_{0} a_{1}+\left(b-a_{2}\right)\left(a_{2}-a_{1}\right) .
$$


In addition, for any $p \in \mathbb{Z} \backslash\{0, \pm 1\}$, the estimates

$$
|F(\boldsymbol{a} ; b)|=|p|^{O(b)}, \quad|A(p)| \leq|p|^{\left(a_{0}+a_{1}+a_{2}\right) b-\left(a_{1}^{2}+a_{2}^{2}+b^{2}\right) / 2+O(b)}
$$

hold with some absolute constant in $O(b)$.

Proof. The first condition in (14) allows us to write $a_{1}^{*}=a_{1}, a_{2}^{*}=a_{2}$ in (10), (11), and to apply Lemma 3 from [Zu1] to the quantity $B_{2}(p)$ after interchanging the summations in (11):

$$
\begin{aligned}
B_{2}(p)= & p^{a_{1}\left(a_{1}-1\right) / 2+a_{0} a_{1}+\left(b-a_{2}\right)\left(a_{2}-a_{1}\right)} \sum_{j=1}^{a_{0}-1} \frac{1}{p^{j}-1} \\
& \times \sum_{l=0}^{a_{2}-1}(-1)^{l} p^{\left(a_{1}-l\right)\left(a_{1}-l-1\right) / 2} \\
& \times\left[\begin{array}{c}
b-a_{2}+l-1 \\
a_{1}-1
\end{array}\right]_{p}\left[\begin{array}{c}
a_{2}-1 \\
l
\end{array}\right]_{p}\left(p^{a_{0}-j-1} ; p^{-1}\right)_{b-a_{1}-a_{2}+l} .
\end{aligned}
$$

From this formula we deduce that

$$
p^{-M_{2}} D_{a_{0}-1}(p) B_{2}(p) \in \mathbb{Z}[p],
$$

where $M_{2}=a_{1}\left(a_{1}-1\right) / 2+a_{0} a_{1}+\left(b-a_{2}\right)\left(a_{2}-a_{1}\right)$, while formulae (8), (10) and the inequality

$$
\frac{(b-k)(b-k-1)}{2}+a_{0} k \geq \frac{\left(b-a_{2}\right)\left(b-a_{2}-1\right)}{2}+a_{0} a_{2}, \quad k \geq a_{2} \geq b-a_{0}
$$

yield

$$
p^{-M_{1}}(p) A(p) \in \mathbb{Z}[p], \quad p^{-M_{1}} D_{b-a_{1}-1}(p) B_{1}(p) \in \mathbb{Z}[p],
$$

where $M_{1}=a_{1}\left(a_{1}-1\right) / 2+a_{0} a_{2}$. Thus using the fact that $\min \left\{M_{1}, M_{2}\right\}=$ $M_{2}$ under the hypothesis (14), we arrive at (15).

To prove the second part of the lemma, we adopt the construction and results of [Zu1]. There we consider the family of series

$$
\begin{aligned}
I(\boldsymbol{n} ; m)= & I\left(n_{0}, n_{1}, n_{2} ; m\right) \\
= & \frac{(q ; q)_{n_{2}}}{(q ; q)_{n_{0}}} q^{-n_{0}\left(n_{1}-n_{0}\right)} \\
& \times \sum_{s=n_{1}+1}^{\infty} \frac{\left(1-q^{n_{1}-n_{0}+1-s}\right) \ldots\left(1-q^{n_{1}-s}\right)}{\left(q^{-s}-1\right)\left(q^{-s}-q\right) \ldots\left(q^{-s}-q^{n_{2}}\right)} q^{(m-1) s} \\
= & (-1)^{n_{0}} q^{n_{0}\left(n_{0}+1\right) / 2+\left(n_{1}+1\right)\left(n_{2}-n_{0}+m\right)} \\
& \times \frac{(q ; q)_{n_{1}}(q ; q)_{n_{2}}}{(q ; q)_{n_{1}+n_{2}+1}} \cdot{ }_{2} \phi_{1}\left(\begin{array}{c}
q^{n_{0}+1}, q^{n_{1}+1} \\
q^{n_{1}+n_{2}+2}
\end{array} \mid q, q^{n_{2}-n_{0}+m}\right),
\end{aligned}
$$

where $n_{0}, n_{1}, n_{2}$, and $m$ are positive integers satisfying the conditions

$$
n_{1} \geq n_{0}, \quad n_{2} \geq n_{0}, \quad m>n_{0} .
$$


Comparing representations (13) and (18) we conclude that

$$
F(\boldsymbol{a} ; b)=(-1)^{n_{0}} p^{n_{0}\left(n_{0}+1\right) / 2+\left(n_{1}+1\right)\left(n_{2}-n_{0}+m\right)} I(\boldsymbol{n} ; m),
$$

where

$$
n_{0}=a_{1}-1, \quad n_{1}=a_{2}-1, \quad n_{2}=b-a_{2}-1, \quad m=a_{0}+a_{1}+a_{2}-b,
$$

and conditions (19) become (14). Therefore the estimates (17) are consequences of the corresponding results for the quantity $I(\boldsymbol{n} ; m)$ (see [Zu1, Lemmas 6 and 7]), and the lemma follows.

REMARK. In [Zu1] we made a mistake in computing the exponent of $p$ when applying the identity of [Zu1, Lemma 3]. The correct application of the identity leads to

$$
\begin{aligned}
p^{\left(n_{1}-n_{0}\right)\left(m-n_{0}+1\right)} D_{\max \left\{n_{1}+n_{2}-n_{0}, m\right\}} \cdot I & \in \mathbb{Z}[p] h_{p}(1)+\mathbb{Z}[p], \\
p^{\left(n_{1}-n_{0}\right)\left(m-n_{0}+1\right)} \widehat{D}_{n_{1}+n_{2}-n_{0}, m} \cdot \widehat{I} & \in \mathbb{Z}[p] \ln _{p}(2)+\mathbb{Z}[p],
\end{aligned}
$$

and to the corresponding changes in Propositions 1, 2 of [Zu1]. Fortunately, these changes do not influence the result of Theorem 2 of [Zu1] (concerning the irrationality exponent of $\ln _{p}(2)$ ), and Theorem 1 in the present work considerably improves the wrong result of Theorem 1 of [Zu1].

4. Permutation group for $q$-harmonic series. Heine's transform (1) yields the stability of the quantity

$$
\begin{aligned}
& \frac{F\left(a_{0}, a_{1}, a_{2} ; b\right)}{\Gamma_{q}\left(a_{0}\right) \Gamma_{q}\left(a_{2}\right) \Gamma_{q}\left(b-a_{2}\right)} \\
& =\frac{1}{(1-q) \Gamma_{q}\left(a_{0}\right) \Gamma_{q}(b)} \cdot{ }_{2} \phi_{1}\left(\begin{array}{c|c}
q^{a_{1}}, q^{a_{2}} & q, q^{a_{0}} \\
q^{b} & \mid
\end{array}\right)
\end{aligned}
$$

under the action of

$$
\tau:\left(a_{0}, a_{1}, a_{2} ; b\right) \mapsto\left(a_{1}, b-a_{1}, a_{0} ; a_{0}+a_{2}\right) .
$$

In addition, the quantity (20) is obviously stable under the action of the permutation

$$
\sigma:\left(a_{0}, a_{1}, a_{2} ; b\right) \mapsto\left(a_{0}, a_{2}, a_{1} ; b\right)
$$

interchanging the parameters $a_{1}$ and $a_{2}$. Let $\mathfrak{G}$ denote the group generated by $\tau, \sigma$; the group $\mathfrak{G}=\left\langle\tau, \sigma: \tau^{6}=\sigma^{2}=(\tau \sigma)^{2}=\mathrm{id}\right\rangle$ has order 12 (see [Fi, Section 20]).

To interpret $\mathfrak{G}$ as a permutation group, we now introduce the tuple $\boldsymbol{c}$ of the six additional parameters

$$
\begin{gathered}
c_{00}=a_{0}+a_{1}+a_{2}-b-1, \quad c_{01}=a_{0}-1, \quad c_{11}=a_{1}-1, \\
c_{21}=a_{2}-1, \quad c_{12}=b-a_{1}-1, \quad c_{22}=b-a_{2}-1,
\end{gathered}
$$


and take $H(\boldsymbol{c}):=F(\boldsymbol{a} ; b)$. Then

$$
\tau=\left(\begin{array}{llllll}
c_{22} & c_{21} & c_{01} & c_{11} & c_{12} & c_{00}
\end{array}\right), \quad \sigma=\left(\begin{array}{lll}
c_{11} & c_{21}
\end{array}\right)\left(\begin{array}{lll}
c_{12} & c_{22}
\end{array}\right)
$$

are permutations of the tuple $\boldsymbol{c}$ of orders 6 and 2, respectively, and the $\mathfrak{G}$ stability of the quantity (20) can be summarized by the following assertion.

LEMma 5. The quantity

$$
\frac{H(\boldsymbol{c})}{\Pi_{q}(\boldsymbol{c})}, \quad \text { where } \quad \Pi_{q}(\boldsymbol{c}):=\left[c_{01}\right]_{q} !\left[c_{21}\right]_{q} !\left[c_{22}\right]_{q} !,
$$

is stable under the action of $\mathfrak{G}$.

By definition of the $q$-factorial coefficient, $\Pi_{q}(\boldsymbol{c})=p^{-N(\boldsymbol{c})} \Pi_{p}(\boldsymbol{c})$, where

$$
N(\boldsymbol{c}):=\frac{c_{01}\left(c_{01}+1\right)+c_{21}\left(c_{21}+1\right)+c_{22}\left(c_{22}+1\right)}{2} .
$$

We also require some additional characteristics: $M(\boldsymbol{c}):=M(\boldsymbol{a} ; b)$ is the "suitable" integer of Lemma 3 if $a_{1}+a_{2} \leq b$ or is determined by formula (16) if stronger conditions (14) hold, and

$$
\begin{aligned}
m(\boldsymbol{c}) & :=\max \left\{c_{00}, c_{01}, c_{11}, c_{21}, c_{12}, c_{22}\right\}, \\
s_{+}(\boldsymbol{c}) & :=c_{01}+c_{21}+c_{22}=a_{0}+b-3, \\
s_{-}(\boldsymbol{c}) & :=c_{00}+c_{11}+c_{12}=a_{0}+a_{1}+a_{2}-3, \\
s(\boldsymbol{c}) & :=s_{+}(\boldsymbol{c})-s_{-}(\boldsymbol{c})=b-a_{1}-a_{2} .
\end{aligned}
$$

Then $m(\boldsymbol{c})$ is $\mathfrak{G}$-stable, while the quantities $s_{ \pm}(\boldsymbol{c})$ and $s(\boldsymbol{c})$ obey the following rules.

Lemma 6. The following relations hold:

$$
\begin{array}{lll}
s_{+}(\tau \boldsymbol{c})=s_{-}(\boldsymbol{c}), & s_{-}(\tau \boldsymbol{c})=s_{+}(\boldsymbol{c}), & s(\tau \boldsymbol{c})=-s(\boldsymbol{c}), \\
s_{+}(\sigma \boldsymbol{c})=s_{+}(\boldsymbol{c}), & s_{-}(\sigma \boldsymbol{c})=s_{-}(\boldsymbol{c}), & s(\sigma \boldsymbol{c})=s(\boldsymbol{c}),
\end{array}
$$

where $\mathfrak{g c}$ denotes the image of $\boldsymbol{c}$ under the action of a permutation $\mathfrak{g} \in \mathfrak{G}$.

Proof. By direct computation.

As is easily seen, condition (6) is equivalent to $s(\boldsymbol{c}) \geq 0$; hence we can write the conclusions of Lemmas 3 and 4 in the form

$$
p^{-M(\boldsymbol{c})} D_{m(\boldsymbol{c})}(p) H(\boldsymbol{c}) \in \mathbb{Z}[p] h_{p}(1)+\mathbb{Z}[p],
$$

if (and only if) $s(\boldsymbol{c}) \geq 0$. This fact and Lemma 6 mean that we might use the group $\mathfrak{G}_{+}=\left\langle\tau^{2}, \sigma\right\rangle \subset \mathfrak{G}$ of order 6 instead of the total group $\mathfrak{G}$ if $s(\boldsymbol{c})>0$. The case $s(\boldsymbol{c})=0$ is of no interest since it implies the relations $c_{00}=c_{01}$, $c_{11}=c_{22}, c_{21}=c_{12}$, and as a consequence

$$
\Pi_{p}(\mathfrak{g} \boldsymbol{c})=\Pi_{p}(\boldsymbol{c}) \quad \text { for all } \mathfrak{g} \in \mathfrak{G} .
$$


If $s(\boldsymbol{c})>0$, we obtain at most three different values of $\Pi_{p}(\mathfrak{g} \boldsymbol{c}), \mathfrak{g} \in \mathfrak{G}_{+}$, namely,

$$
\begin{aligned}
& \Pi_{p}=\Pi_{p}(\boldsymbol{c})=\Pi_{p}\left(\sigma \tau^{4} \boldsymbol{c}\right)=\left[c_{01}\right]_{p} !\left[c_{21}\right]_{p} !\left[c_{22}\right]_{p} ! \\
& \Pi_{p}^{\prime}=\Pi_{p}\left(\tau^{2} \boldsymbol{c}\right)=\Pi_{p}(\sigma \boldsymbol{c})=\left[c_{01}\right]_{p} !\left[c_{11}\right]_{p} !\left[c_{12}\right]_{p} ! \\
& \Pi_{p}^{\prime \prime}=\Pi_{p}\left(\tau^{4} \boldsymbol{c}\right)=\Pi_{p}\left(\sigma \tau^{2} \boldsymbol{c}\right)=\left[c_{00}\right]_{p} !\left[c_{12}\right]_{p} !\left[c_{22}\right]_{p} !
\end{aligned}
$$

For each $l=2,3, \ldots, m(\boldsymbol{c})$, take

$$
\begin{aligned}
\nu_{l} & :=\max _{\mathfrak{g} \in \mathfrak{G}_{+}} \operatorname{ord}_{\Phi_{l}(p)} \frac{\Pi_{p}(\boldsymbol{c})}{\Pi_{p}(\mathfrak{g} \boldsymbol{c})} \\
& =\max \left\{0,\left\lfloor\frac{c_{21}}{l}\right\rfloor+\left\lfloor\frac{c_{22}}{l}\right\rfloor-\left\lfloor\frac{c_{11}}{l}\right\rfloor-\left\lfloor\frac{c_{12}}{l}\right\rfloor,\right. \\
& \left.\left\lfloor\frac{c_{01}}{l}\right\rfloor+\left\lfloor\frac{c_{21}}{l}\right\rfloor-\left\lfloor\frac{c_{00}}{l}\right\rfloor-\left\lfloor\frac{c_{12}}{l}\right\rfloor\right\},
\end{aligned}
$$

and set

$$
\Omega(p):=\prod_{l=2}^{m(c)} \Phi_{l}^{\nu_{l}}(p) \in \mathbb{Z}[p] .
$$

Lemma 7. We have

$$
p^{-M(\boldsymbol{c})} D_{m(\boldsymbol{c})}(p) \Omega^{-1}(p) H(\boldsymbol{c}) \in \mathbb{Z}[p] h_{p}(1)+\mathbb{Z}[p],
$$

provided that $s(\boldsymbol{c})>0$.

Proof. We follow the lines of the proof of Proposition 2 in [Zu2]. The inclusion (21) and Lemma 5 yield

$$
\begin{aligned}
p^{-M(\mathfrak{g} c)-N(\mathfrak{g} c)+N(\boldsymbol{c})} D_{m(\boldsymbol{c})} & (p) \cdot \frac{\Pi_{p}(\mathfrak{g} \boldsymbol{c})}{\Pi_{p}(\boldsymbol{c})} \cdot H(\boldsymbol{c}) \\
= & p^{-M(\mathfrak{g} c)} D_{m(\mathfrak{g} c)}(p) H(\mathfrak{g} c) \in \mathbb{Z}[p] h_{p}(1)+\mathbb{Z}[p]
\end{aligned}
$$

for all $\mathfrak{g} \in \mathfrak{G}_{+}$. Since cyclotomic polynomials enter the $p$-factorial $[n]_{p}$ ! in accordance with formula (5) and these polynomials are coprime to the polynomial $p \in \mathbb{Z}[p]$, we arrive at $(23)$.

5. $q$-Conclusion. We now take a tuple of new positive integers (directions) $\alpha_{0}, \alpha_{1}, \alpha_{2}$, and $\beta$ satisfying the conditions

$$
\alpha_{1} \leq \alpha_{2}, \quad \alpha_{1}+\alpha_{2}<\beta \leq \alpha_{0}+\alpha_{2},
$$

and to each integer $n=1,2, \ldots$ assign the old parameters $\boldsymbol{a}$ and $b$ in accordance with the following rule:

$$
a_{j}=\alpha_{j} n+1, \quad j=0,1,2, \quad b=\beta n+2 .
$$

Then setting

$$
c_{00}=\alpha_{0}+\alpha_{1}+\alpha_{2}-\beta, \quad c_{j 1}=\alpha_{j}, j=0,1,2, \quad c_{j 2}=\beta-\alpha_{j}, j=1,2,
$$


and $m=m(\boldsymbol{c})$ we introduce the quantities

$$
H_{n}:=H(\boldsymbol{c} \cdot n)=F(\boldsymbol{a} ; b)=A_{n} h_{p}(1)-B_{n}, \quad n=1,2, \ldots
$$

Finally,

$$
\begin{array}{r}
p^{-M(a ; b)} D_{m n}(p) \Omega^{-1}(p) H_{n} \in \mathbb{Z}[p] h_{p}(1)+\mathbb{Z}[p] \subset \mathbb{Z} h_{p}(1)+\mathbb{Z}, \\
n=1,2, \ldots,
\end{array}
$$

by Lemma 7, and

$$
\begin{gathered}
\lim _{n \rightarrow \infty} \frac{\log \left|H_{n}\right|}{n^{2} \log |p|}=0, \\
\limsup _{n \rightarrow \infty} \frac{\log \left|A_{n}\right|}{n^{2} \log |p|} \leq\left(\alpha_{0}+\alpha_{1}+\alpha_{2}\right) \beta-\frac{\alpha_{1}^{2}+\alpha_{2}^{2}+\beta^{2}}{2}=: C_{1}
\end{gathered}
$$

by Lemma 4 . In addition, $\nu_{l}=\omega(n / l)$ in (22), where

$$
\begin{aligned}
\omega(x):=\max \left\{0,\left\lfloor c_{21} x\right\rfloor+\left\lfloor c_{22} x\right\rfloor-\right. & \left\lfloor c_{11} x\right\rfloor-\left\lfloor c_{12} x\right\rfloor, \\
& \left.\left\lfloor c_{01} x\right\rfloor+\left\lfloor c_{21} x\right\rfloor-\left\lfloor c_{00} x\right\rfloor-\left\lfloor c_{12} x\right\rfloor\right\}
\end{aligned}
$$

is a 1-periodic integer-valued function; therefore,

$$
\begin{aligned}
\lim _{n \rightarrow \infty} \frac{\log \left|p^{M(a ; b)} D_{m n}^{-1}(p) \Omega(p)\right|}{n^{2} \log |p|} \\
=\frac{1}{2} \alpha_{1}^{2}+\alpha_{0} \alpha_{1}+\left(\beta-\alpha_{2}\right)\left(\alpha_{2}-\alpha_{1}\right) \\
\quad-\frac{3}{\pi^{2}}\left(m^{2}-\int_{0}^{1} \omega(x) d\left(-\psi^{\prime}(x)\right)\right)=: C_{0}
\end{aligned}
$$

by Lemmas 1, 2, and 4. By standard arguments, relations (24)-(26) yield the estimate $\mu\left(h_{p}(1)\right) \leq C_{1} / C_{0}$, provided that $C_{0}>0$.

Take

$$
\alpha_{0}=14, \quad \alpha_{1}=12, \quad \alpha_{2}=14, \quad \beta=27
$$

so that

$$
c_{00}=13, \quad c_{01}=14, \quad c_{11}=12, \quad c_{21}=14, \quad c_{12}=15, \quad c_{22}=13 .
$$

Then

$$
C_{1}=545.5, \quad C_{0}=221.30008816 \ldots,
$$

hence $\mu\left(h_{p}(1)\right) \leq C_{1} / C_{0}=2.46497868 \ldots$ Here $\omega(x)=1$ for $x \in[0,1)$ belonging to the set

$$
\begin{aligned}
{\left[\frac{1}{14}, \frac{1}{12}\right) \cup\left[\frac{1}{7}, \frac{1}{6}\right) \cup\left[\frac{3}{14}, \frac{1}{4}\right) \cup\left[\frac{2}{7}, \frac{1}{3}\right) \cup\left[\frac{5}{14}, \frac{2}{5}\right) \cup\left[\frac{3}{7}, \frac{7}{15}\right) \cup\left[\frac{1}{2}, \frac{8}{15}\right) } \\
\cup\left[\frac{4}{7}, \frac{3}{5}\right) \cup\left[\frac{9}{14}, \frac{2}{3}\right) \cup\left[\frac{5}{7}, \frac{11}{15}\right) \cup\left[\frac{11}{14}, \frac{4}{5}\right) \cup\left[\frac{6}{7}, \frac{13}{15}\right) \cup\left[\frac{13}{14}, \frac{14}{15}\right) .
\end{aligned}
$$

The proof of Theorem 1 is complete. 
REMARK. The series (13) involving linear forms in 1 and the $q$-harmonic series can be represented as some $q$-integrals (see, e.g., [Ex, Section 2.5.1]). This $q$-integral representation is very similar to that used in [RV1] and [RV2] for describing the permutation groups for $\zeta(2)$ and $\zeta(3)$. In spite of this similarity, there exists no general pattern to change the variable of $q$-integration (see [Ask] and [Ex, Section 2.2.4]). Therefore the hypergeometric construction proposed in this paper as well as in our previous works [Zu1], [Zu2] looks a very natural way to extend the group-structure approach to solving new number-theoretic problems.

Acknowledgements. The grounds of this work were set when the author was a visitor of the Department of Mathematical Sciences, University of Oulu (25 March-6 April 2002). I would like to express my deep gratitude to the department for their kind hospitality.

\section{References}

[Ask] R. Askey, The q-gamma and q-beta functions, Appl. Anal. 8 (1978), 125-141.

[Ass] W. Van Assche, Little q-Legendre polynomials and irrationality of certain Lambert series, Ramanujan J. 5 (2001), 295-310.

[Bé] J.-P. Bézivin, Indépendence linéaire des valeurs des solutions transcendantes de certaines équations fonctionnelles, Manuscripta Math. 61 (1988), 103-129.

[Bo] P. Borwein, On the irrationality of $\sum \frac{1}{q^{n}+r}$, J. Number Theory 37 (1991), 253-259.

[BV] P. Bundschuh and K. Väänänen, Arithmetical investigations of a certain infinite product, Compositio Math. 91 (1994), 175-199.

[Er] P. Erdős, On arithmetical properties of Lambert series, J. Indian Math. Soc. 12 (1948), 63-66.

[Ex] H. Exton, q-Hypergeometric Functions and Applications, Ellis Horwood Ser. Math. Appl., Ellis Horwood, Chichester, 1983.

[Fi] N. J. Fine, Basic Hypergeometric Series and Applications, Math. Surveys Monogr. 27, Amer. Math. Soc., Providence, RI, 1988.

[GR] G. Gasper and M. Rahman, Basic Hypergeometric Series, Encyclopedia Math. Appl. 35, Cambridge Univ. Press, Cambridge, 1990.

[He1] E. Heine, Über die Reihe

$$
1+\frac{\left(q^{\alpha}-1\right)\left(q^{\beta}-1\right)}{(q-1)\left(q^{\gamma}-1\right)} x+\frac{\left(q^{\alpha}-1\right)\left(q^{\alpha+1}-1\right)\left(q^{\beta}-1\right)\left(q^{\beta+1}-1\right)}{(q-1)\left(q^{2}-1\right)\left(q^{\gamma}-1\right)\left(q^{\gamma+1}-1\right)} x^{2}+\ldots,
$$

J. Reine Angew. Math. 32 (1846), 210-212.

[He2] -, Untersuchungen über die Reihe

$$
1+\frac{\left(q^{\alpha}-1\right)\left(q^{\beta}-1\right)}{(q-1)\left(q^{\gamma}-1\right)} x+\frac{\left(q^{\alpha}-1\right)\left(q^{\alpha+1}-1\right)\left(q^{\beta}-1\right)\left(q^{\beta+1}-1\right)}{(q-1)\left(q^{2}-1\right)\left(q^{\gamma}-1\right)\left(q^{\gamma+1}-1\right)} x^{2}+\ldots,
$$

ibid. 34 (1847), 285-328.

[MV] T. Matala-Aho and K. Väänänen, On approximation measures of q-logarithms, Bull. Austral. Math. Soc. 58 (1998), 15-31. 
[RV1] G. Rhin and C. Viola, On a permutation group related to $\zeta(2)$, Acta Arith. 77 (1996), 23-56.

[RV2] -, - , The group structure for $\zeta(3)$, ibid. 97 (2001), 269-293.

[Zu1] W. Zudilin, Remarks on irrationality of q-harmonic series, Manuscripta Math. 107 (2002), 463-477.

[Zu2] - On the irrationality measure for a q-analogue of $\zeta(2)$, Mat. Sb. 193 (2002), no. 8, 49-70 (in Russian); English transl.: Sb. Math. 193 (2002), 1151-1172.

Department of Mechanics and Mathematics

Moscow Lomonosov State University

Vorobiovy Gory, GSP-2

119992 Moscow, Russia

E-mail: wadim@ips.ras.ru

URL: http://wain.mi.ras.ru/index.html

Received on 13.5.2002

and in revised form on 10.4.2003 